\title{
Convergence Conditions for Random Quantum Circuits
}

\author{
Joseph Emerson* ${ }^{1,2}$ Etera Livine $^{\dagger},{ }^{2}$ and Seth Lloyd ${ }^{\ddagger 3}$ \\ ${ }^{1}$ Institute for Quantum Computing and Dept of Applied Math, University of Waterloo \\ ${ }^{2}$ Perimeter Institute for Theoretical Physics \\ ${ }^{3}$ MIT, Dept of Mechanical Engineering
}

(Dated: October 16, 2018)

\begin{abstract}
Efficient methods for generating pseudo-randomly distributed unitary operators are needed for the practical application of Haar distributed random operators in quantum communication and noise estimation protocols. We develop a theoretical framework for analyzing pseudo-random ensembles generated through a random circuit composition. We prove that the measure over random circuits converges exponentially (with increasing circuit length) to the uniform (Haar) measure on the unitary group, though the rate for uniform convergence must decrease exponentially with the number of qubits. We describe how the rate of convergence for test functions associated with specific randomization tasks leads to weaker convergence conditions which may allow efficient random circuit constructions.
\end{abstract}

Random unitary operators, consisting of unitary operators drawn randomly from the invariant (Haar) measure on $U(D)$, comprise a powerful resource for quantum computation and quantum communication. Recent work has shown that random unitary operators enable efficient protocols for characterizing noise (decoherence) on universal quantum processing devices [1, 2, 3$]$. Noise estimation is an important problem for the experimental development of coherent quantum control [4, 5] and, ultimately, fault-tolerant quantum processing via the optimization of error-correction schemes [6]. Moreover, random unitary operators can be applied to randomize arbitrary quantum state and consequently have proven useful for a number of quantum communication protocols, including approximate quantum encryption [7], remote state preparation [8], and the superdense coding of quantum states [9]. A practical drawback for the above applications is that a decomposition of the Haar-distributed random operators in terms of one and two qubit gates (which is required to generate a random unitary operator on a quantum processor) requires quantum circuits of exponential length $\mathcal{O}\left(D^{2} \log (D)^{3}\right)$ 10. Such circuits are termed inefficient because the length grows exponentially with the number of qubits $\left(\log _{2}(D)\right)$.

In Ref. 1], families of random circuits, i.e., circuits that are composed of sequences of quantum gates drawn independently and randomly from some universal gate set, were proposed as a means of generating a set of pseudo-random unitary operators, i.e., a set that is for certain practical purposes indistinguishable from a Haardistributed set of random unitary operators. The possibility that pseudo-random sets may be generated efficiently on a quantum computer is suggested from previous work in quantum chaos, which has shown that quan-

\footnotetext{
*jemerson@perimeterinstitute.ca

†elivine@perimeterinstitute.ca

${ }^{\ddagger}$ slloyd@mit.edu
}

tum chaos models reproduce relevant characteristics of random unitary operators [1] even though these systems may be simulated with efficient quantum circuits 12]. Moreover, numerical studies have shown that random circuits of efficient length can reproduce that Haar distribution of subsystem purity [1, 13], and experimental evidence indicates that sufficiently random operators can be generated with the currently available levels of experimental control with NMR quantum processors [1, 14].

In this paper we develop a set of mathematical tools that can be applied to analyze measures over random circuits. We apply these tools to demonstrate that under increasing length of the random circuit the measure over random circuits converges uniformly to the Haar measure on the group. We show that the rate of convergence (as a function of increasing gate depth) is generically exponential, though the exponent must itself decrease exponentially with the number of qubits. At the end of this paper we describe how specific applications can define weaker convergence conditions, leading to the possibility that efficient random circuits composed from a discrete set of universal gates might be able to adequately mimic the Haar measure.

We now prove that the measure of a product of unitary operators, with each factor in the product drawn independently from a measure enjoying support on a subset of $U(D)$ that generates the full group (viz, a continuous universal gate set) or a dense subset of the full group (viz, a discrete universal gate set), converges exponentially to the constant function with respect to the Haar measure on the group. Let $G$ denote the (compact Lie) group $U(D)$ with elements $g \in G$. Let $\mathcal{F}$ denote the set of probability measures over this group. Suppose the elements $g_{1}$ and $g_{2}$ are drawn at random from the measures $f_{1} \in \mathcal{F}$ and $f_{2} \in \mathcal{F}$ respectively. The composed element $g=g_{1} \cdot g_{2}$, where $\cdot$ denotes the standard group multiplication, is then distributed according to

$$
f(g)=\left(f_{1} * f_{2}\right)(g)=\int d \mu(h) f_{1}\left(g h^{-1}\right) f_{2}(h),
$$


where $*$ denotes the convolution product and $d \mu(g)$ denotes the Haar measure on $G$. The convolution operator for $m$ convolution factors is denoted $f^{* m} \equiv f * f^{*(m-1)}$. Assuming that the initial function $f$ is a probability measure then all its convolution powers $f^{* m}$ are also automatically probability measures, and hence have the properties,

$$
\begin{aligned}
\forall g, \quad f^{* m}(g) & \geq 0 \\
\int_{G} d \mu(g) f^{* m}(g) & =1 .
\end{aligned}
$$

We are interested in the convergence properties of the sequence of probability distributions $f^{* m}$ as $m \rightarrow \infty$. More precisely, we would like to prove its convergence to the uniform function with respect to the Haar measure and obtain an estimate of its rate of convergence. As an aside let us first describe the intuition for expecting convergence to the Haar measure. We note that only the characters of the group are stable under convolution [15]. Therefore, if $f^{* m}$ converges at all then it must converge to a character of the group. Moreover it must converge to the Haar measure because this is the only character which is everywhere non-negative. The hard part is now to determine under what conditions the sequence $f^{* m}$ actually converges.

An important tool in the study of convolution products is the Fourier decomposition. Indeed we will make use of the fact that the convolution product turns into a simple standard product for the Fourier components. In the case of compact Lie groups, a Fourier representation of functions is provided by the Peter-Weyl theorem (see e.g. [16]). Let $\hat{G}=\left\{T^{s}\right\}$ be the set of non-equivalent irreducible unitary representations of $G$. We order the irreducible representations $s$ by increasing dimensionality $d_{s}$, where the trivial representation has dimensionality $d_{0}=1$. The functions $\sqrt{d_{s}} D_{j k}^{s}$ (taking $g \rightarrow \mathbb{C}$ ) for $s \in \hat{G}$ and $1 \leq j, k \leq d_{s}$, where $D_{j k}^{s}(g)$ are the matrix elements of $T^{s}$, form a complete orthonormal set for the Hilbert space $L^{2}(G)$ of square-integrable functions on $G$ with respect to the Haar measure. The orthogonality relations read:

$$
\int_{G} d \mu(g) D_{i j}^{s}(g) \overline{D_{m n}^{s^{\prime}}(g)}=\frac{\delta^{s s^{\prime}} \delta_{i m} \delta_{j n}}{d_{s}}
$$

where $\delta^{s s^{\prime}}$ is 1 if $T^{s}$ and $T^{s^{\prime}}$ are equivalent and zero otherwise. Then, any function $f \in L^{2}(G)$ can be decomposed as,

$$
f(g)=\sum_{s \in \hat{G}} \sum_{j, k=1}^{d_{s}} \hat{f}_{j k}^{s} D_{j k}^{s}(g),
$$

with Fourier coefficients

$$
\hat{f}_{j k}^{s}=d_{s} \int_{G} d \mu(g) f(g) \overline{D_{j k}^{s}(g)},
$$

and in particular,

$$
\int_{G} d \mu(g) f(g)=\hat{f}^{s=0} .
$$

We have the following integral identity:

$$
\int_{G} d \mu(g)|f(g)|^{2}=\sum_{s \in \hat{G}} \frac{1}{d_{s}} \operatorname{tr}\left(\left(\hat{f}^{s}\right)^{\dagger} \hat{f}^{s}\right)<\infty
$$

Finally, the normalized constant function (with respect to the Haar measure) has Fourier coefficients:

$$
\hat{\mu}^{s=0}=1, \quad \hat{\mu}_{j \bar{k}}^{s \geq 1}=0 .
$$

In general the Fourier transform of the convolution product has a coefficient matrix for each irreducible representation of the group given by the product of the coefficient matrices from the same irreducible representation of the Fourier transforms of the original convolved functions. That is, given two functions $\phi, \psi$, the Fourier transform of their convolution product is given as:

$$
(\widehat{\phi * \psi})^{s}=\frac{1}{d_{s}} \widehat{\phi}^{s} \widehat{\psi}^{s}
$$

Therefore the convoluted powers of the initial probability distribution reads:

$$
\left(\widehat{f^{* m}}\right)^{s}=d_{s}\left(\frac{\widehat{f} s}{d_{s}}\right)^{m}
$$

where $\left(\widehat{f^{* m}}\right)^{s}$ denotes the $d_{s} \times d_{s}$ matrix of coefficients appearing in the Fourier representation of $f^{* m}$ and $\left(\hat{f}^{s}\right)^{m}$ denotes the $m$ 'th power of the $d_{s} \times d_{s}$ matrix of coefficients appearing in the Fourier representation of the initial distribution $f$. The normalization condition implies $\left(f^{\hat{*} m}\right)^{s=0}=1$.

We assume that the initial measure $f$ is continuous and enjoys support only on some (small) subset of $G$ that generates the full group $G$. This subset might have very small measure compared to the full group; one example is the continuous gate set consisting of all single qubit rotations coupled with a CNOT gate between all qubit pairs (later we relax this assumption and consider convergence conditions for discrete and finite universal gate sets). We have the following lemma: the matrix $\hat{f}^{s}$ has norm strictly less than $d_{s}$ for $s>0$. To prove this we consider the usual vector norm, $|x|_{2} \equiv \sqrt{\langle x \mid x\rangle}$, and observe that for all vectors $x$ in the $s$ representation, we have [18]:

$$
\begin{aligned}
\left|\hat{f}^{s} x\right|_{2} & =\left|d_{s} \int_{G} d \mu(g) f(g) \bar{D}^{(s)}(g) x\right|_{2} \\
& \leq d_{s} \int_{G} d \mu(g)\left|f(g) \bar{D}^{(s)}(g) x\right|_{2} \\
& \leq d_{s} \int_{G} d \mu(g) f(g)\left\|\bar{D}^{(s)}(g)\right\||x|_{2}=d_{s}|x|_{2}
\end{aligned}
$$


where we have introduced the norm, $\|D\| \equiv$ $\max _{x \neq 0}|D x|_{2} /|x|_{2}$, and used that $\|D\|=1$ since $D(g)$ is unitary. Equality holds if and only if $D(g) x=\xi(g) y$ is true for all $g$ for which $f(g)>0$, where $\xi(g) \in \mathbb{C}$. Since any $g \in G$ can be generated by the support of $f$, $D(g) x=\xi(g) y$ must hold also for all $g \in G$. This implies we have a 1-D representation of $G$ embedded in the irreducible representation $s$. Hence the equality is not saturated unless $s$ is the trivial (identity) representation. It follows that, when $s \neq 0$, i.e. for all $d_{s}>1$, we have $\forall x,\left|\hat{f}^{s} x\right|<d_{s}|x|$, hence

$$
\left\|\hat{f}^{s}\right\| \equiv \max _{x \neq 0} \frac{\left|\hat{f}^{s} x\right|_{2}}{|x|_{2}}=\max _{|x|_{2}=1}\left|\hat{f}^{s} x\right|_{2}<d_{s} .
$$

Our proof is not concluded: this bound does not guarantee uniform convergence to the uniform measure (given by Eqs. (17) because the Fourier representation for generic functions involves an infinite sum, and hence the eigenvalues of very "high frequency" Fourier modes can a priori come arbitrarily close to 1 . Nevertheless, a first remark is that polynomial functions $f$ (technically polynomials in the matrix elements of $g$ ) are described by a finite sum in the Peter-Weyl decomposition. For such a case, denoting by $S$ the "frequency cutoff" from the maximal representation label for the given polynomial, we obtain the bound

$$
\left\|\left(\hat{f}^{* m}\right)^{s}\right\| \leq d_{s} \alpha^{m}, \quad \text { with } \quad \alpha \equiv \max _{1 \leq s \leq S}\left(\frac{\left\|\hat{f}^{s}\right\|}{d_{s}}\right)<1,
$$

and, consequently, a proof of the exponential convergence of the convoluted powers towards the uniform measure.

For $f$ more generally any continuous function, one can repeat a similar analysis which guarantees a uniform approximation using only a finite Fourier sum. Indeed for $f$ continuous and any $\epsilon>0$ there exists a finite $N_{\epsilon}$ (independent of $g$ ) such that, for all $g \in G$ [16],

$$
\left|f(g)-\sum_{s=1}^{N_{\epsilon}} \sum_{j, k=1}^{d_{s}} \hat{f}_{j k}^{s} D_{j k}^{s}(g)\right| \leq \epsilon .
$$

Let us call $f_{N_{\epsilon}}$ the truncated function with the representation cut-off $N_{\epsilon}$. Then for all $g \in G$ and $m \geq 2$, we use the triangle inequality to write:

$$
\begin{aligned}
\left|f^{* m}(g)-1\right| & \leq\left|f^{* m}(g)-f_{N_{\epsilon}}^{* m}(g)\right|+\left|f_{N_{\epsilon}}^{* m}(g)-1\right| \\
& \leq\left|f^{* m}(g)-f_{N_{\epsilon}}^{* m}(g)\right| \\
& +\left|\sum_{s>1}^{N_{\epsilon}} \sum_{j, k=1}^{d_{s}} d_{s}^{1-m}\left(\left(\hat{f}^{s}\right)^{m}\right)_{j k} D_{j k}^{s}(g)\right| .
\end{aligned}
$$

It is straightforward to bound the first term using the fact that $f_{N_{\epsilon}} * f_{N_{\epsilon}}=f * f_{N_{\epsilon}}$. For all $g \in G$ we have,

$$
\begin{aligned}
\mid f^{* m}(g) & -f_{N_{\epsilon}}^{* m}(g)|=| f *\left(f^{*(m-1)}-f_{N_{\epsilon}}^{*(m-1)}\right)(g) \mid \\
& \leq \int d \mu(h)\left|f\left(g h^{-1}\right) \|\left(f^{*(m-1)}-f_{N_{\epsilon}}^{*(m-1)}\right)(h)\right| \\
& \leq\left\|f^{*(m-1)}-f_{N_{\epsilon}}^{*(m-1)}\right\|_{\infty} \\
& \leq \cdots \leq\left\|f-f_{N_{\epsilon}}\right\|_{\infty} \leq \epsilon,
\end{aligned}
$$

using the fact that $f$ is a positive function with integral equal to 1 . We bound the second term with the help of the inequality:

$$
\left|\operatorname{tr}\left(\left(\hat{f}^{s}\right)^{m} D^{s}(g)\right)\right| \leq d_{s}\left\|\left(\hat{f}^{s}\right)^{m}\right\| \leq d_{s}\left\|\hat{f}^{s}\right\|^{m},
$$

where we use that the vectors of the unitary matrix $D^{s}(g)$ have norm one. It directly follows that:

$$
\left|f_{N_{\epsilon}}^{* m}(g)-1\right| \leq \alpha_{\epsilon}^{m} \sum_{s>1}^{N_{\epsilon}} d_{s}^{2},
$$

where we have defined $\alpha_{\epsilon}=\max _{1<s<N_{\epsilon}}\left(\left\|\hat{f}^{s}\right\| / d_{s}\right)$. As we have shown above, for all non-trivial $s,\left\|\hat{f}^{s}\right\|<d_{s}$ so that $\alpha_{\epsilon}<1$. Therefore there exists a integer $M$, which can be chosen larger than $N_{\epsilon}$, such that $\left|f_{N_{\epsilon}}^{* m}-1\right|_{\infty} \leq \epsilon$ for all $m \geq M$. Then it is obvious that for all $m \geq M$, we have bounded:

$$
\left\|f^{* m}-1\right\|_{\infty} \leq 2 \epsilon .
$$

This concludes the proof that $f^{* m}$ converges uniformly to the constant probability measure on the group for any continuous probability measure. More generally, we claim that the convergence is exponential for any $f \in L^{2}$. Because of Eq. (6) we know that $\operatorname{tr}\left(\left(\hat{f}^{s}\right)^{\dagger} \hat{f}^{s}\right) / d_{s}$ goes to 0 when $s$ goes to $\infty$. Specifically, $\left\|\hat{f}^{s}\right\| \leq \sqrt{\operatorname{tr}\left(\left(\hat{f}^{s}\right)^{\dagger} \hat{f}^{s}\right)}$, and therefore we have $\left\|\hat{f}^{s}\right\| / \sqrt{d_{s}} \rightarrow 0$, so we don't need to worry about the norm of $\hat{f}^{s} / d_{s}$ getting close to 1 when $s \rightarrow \infty$. More precisely, there exists $S_{\delta} \in \mathbb{N}$ such that $\left\|\hat{f}^{s}\right\| / \sqrt{d_{s}} \leq \delta<1$ for all $s>S_{\delta}$. Then for all $s$ we have the bound

$$
\begin{aligned}
\left\|f^{* m}-1\right\|_{\infty} & \leq \sum_{s>0} d_{s}^{-(m-2)}\left\|\hat{f}^{s}\right\|^{m} \\
& \leq \alpha_{\delta}^{m} \sum_{0<s \leq S_{\delta}} d_{s}^{2}+\delta^{m} \sum_{s>S_{\delta}} d_{s}^{-(m / 2-2)}
\end{aligned}
$$

where we have defined,

$$
\alpha_{\delta} \equiv \max _{s \leq S_{\delta}}\left(\frac{\left\|\hat{f}^{s}\right\|}{d_{s}}\right)<1,
$$

As long as $m>6$ the sum over $s$ in the second term converges. Indeed, as explained in [17], the irreducible representations of $U(D)$ are usually labelled by a couple 
of integers $(k, l)$ and their dimension is given in terms of binomial coefficients:

$$
d_{k, l}^{(D)}=\frac{k+l+D-1}{D-1} C_{k+D-2}^{k} C_{l+D-2}^{l} .
$$

It is then straightforward to check that the dimensions grow sufficiently rapidly with $s$ to make the sum over $s$ converge. The exponential $\alpha^{m}$ defines the convergence rate of the convoluted powers of $f$ to the uniform probability measure.

We have shown that the measure over random circuits converges to the Haar measure and moreover that the rate of convergence (with increasing circuit length $m$ ) is exponential. However the exponent will generally depend on the Hilbert space dimension $D$. It is clear that for uniform convergence the exponent must decrease at least as rapidly as $D^{2} \log (D)^{3}$. This follows from the fact that $\mathcal{O}\left(D^{2} \log (D)^{3}\right)$ gates are required to generate all the elements of $U(D)$ from a fixed universal gate set [6]. However, the requirement of uniform convergence we have considered is much stronger than necessary for any practical application. Indeed the practical statistical distinguishability will be limited by practical constraints, such as bounded computational resources or finite sampling from the distribution. In particular we are concerned with convergence with respect to some operationally restricted class of test functions. Hence we demand only that $f^{* m}$ converge to the Haar measure for the "weak topology", i.e. $\int_{G} d \mu(g) f^{* m}(g) \phi(g)$ converges to $\int_{G} d \mu(g) \phi(g)$ for appropriate (continuous) test functions $\phi$. A first example of an operationally motivated test function is the fidelity loss under the motion reversal of a random unitary [3]. Another example comes from the important case of the distinguishability of quantum states evolved under unitary operators drawn from different distributions. Both criteria lead to test functions $\phi$ which are given by polynomials in the matrix elements of the fundamental irreducible (unitary) representation $D^{s=1}(g)$ of $g$, and the finite degree of the polynomial fixes a Fourier cutoff. Hence the convergence is automatically an exponential and the rate of convergence may lead to efficient (scalable) random circuit constructions. Convergence for the weak topology (i.e., for polynomial test functions) also allows us to extend our analysis to arbitrary distributions $f \in L^{1}$, such as finite sums of $\delta$ functions. The same technique applies and we only need to require that $f$ has support on a discrete universal gate set. The argument leading to Eq. 10 still holds and exponential convergence follows for the weak topology, as explained above.

In general then the rate of convergence will depend on the convergence condition specified from an appropriate test function and on the initial probability distribution $f$. The initial distribution $f$ can be modelled in a variety of ways. For example, if we start with a Gaussian packet, then its width will simply increase linearly with the power of the convolution, eventually converging to the uniform measure. The exact convergence rate is then determined directly from the maximum matrix norm of the Fourier components of the initial distribution, where the maximum is taken only over those Fourier components below the cutoff determined from the test function. The appropriate test function (and Fourier cutoff) will generally depend on the specific application. Indeed the numerical analysis of Refs. 1, 13 indicates that for a test function given by the subsystem purity the rate of the exponential convergence is asymptotically independent of the dimension $D$, suggesting that random circuits can generate Haar-distributed subsystem purity efficiently.

We would like to acknowledge R. Cleve, D. Cory, D. Gottesman, J. Goldstone, A. Harrow, H. Pfeiffer, and P. Zanardi for helpful discussions.

[1] J. Emerson, Y. Weinstein, M. Saraceno, S. Lloyd, D. Cory, Pseudo-Random Unitary Operators for Quantum Information Processing, Science 302: 2098-2100 (2003).

[2] J. Emerson, Random Circuits and Pseudo-Random Unitary Operators for Quantum Information Processing, Proceedings of the Seventh International Conference on Quantum Communication, Measurement and Computing, edited by S. M. Barnett, E. Andersson, J. Jeffers, P. Ohberg, O. Hirota (American Institute of Physics), AIP Conf. Proc. 734, 139 (2004); quant-ph/0410087

[3] J. Emerson, R. Alicki, K. Zyczkowski, J. Opt B: Quantum and Semiclassical Optics, 7 S347-S352 (2005).

[4] M. A. Pravia, N. Boulant, J. Emerson, A. Farid, E. Fortunato, T. F. Havel, D. Cory, Robust Control of Quantum Information, J. of Chem. Phys. 119, 19: 9993-10001 (2003); quant-ph/0307062

[5] N. Boulant, S. Furuta, J. Emerson, T. F. Havel, D. Cory, Incoherent Noise and Quantum Control, J. of Chem. Phys. 121, 2955 (2004), quant-ph/0312116

[6] M. Nielsen and I. Chuang, Quantum Computation and Quantum Information, Cambridge (2000).

[7] P. Hayden, D. Leung, P. Shor, A. Winter, Commun. Math. Phys. 250(2):371-391, 2004.

[8] C. H. Bennett, P. Hayden, D. Leung, P. Shor, A. Winter, IEEE Trans. Inform. Theory, vol. 51, no. 1, pp 56-74, 2005.

[9] A. Harrow, P. Hayden, and D. Leung, Phys. Rev. Lett. 92, 187901 (2004).

[10] M. Pozniak, K. Zyczkowski, M. Kus, J. Phys. A, 31, 1059, (1998).

[11] J. Emerson, Y.S. Weinstein, S. Lloyd, D.G. Cory, Phys. Rev. Lett. 89, 284102 (2002).

[12] B. Georgeot, D. L. Shepelyansky, Phys. Rev. Lett. 86, 2890 (2001).

[13] F. Cucchietti and J. Emerson, in preparation.

[14] C. Ryan, J. Emerson, D. Poulin, C. Negrevergne, R. Laflamme, quant-ph/0506085 to appear in Phys. Rev. Lett.

[15] More precisely, if we label the irreducible representations of $G$ by $s$, then the functions $g \mapsto \operatorname{tr}_{s}(g P)$, with $P$ an arbitrary projection on the Hilbert space of the $s$ repre- 
sentation, or any linear combination of such functions (for distinct $s$ 's), are the only functions invariant under the convolution product. The characters are the special case when the projection is trivial $P=\mathrm{Id}_{s}$. Nevertheless, it is still clear that none of these functions are non-negative except for the trivial representation $s=0$.

[16] A.O. Barut and R. Raczka, Theory of Group Representations and Applications, World Scientific (1986); note that in Eq.(7.2.5) the factor of $d_{s}$ should be in the denominator, c.f., Eq[6] above.

[17] N.A. Vilenkin and A.U. Klimyk, Representation of Lie Groups and Special Functions, vols. 1 and 2, Kluwer Academic Publications (1991).

[18] E.J. Hannan, Group Representations and Applied Probability, Methuen and Co. (1965). 